\title{
Cidades inteligentes e cidades sustentáveis: convergência de ações ou mera publicidade?
}

Milton Proença Junior

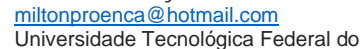
Universidade Tecnológica Federa d
Paraná. Curitiba. Paraná. Brasil.

\section{Rogério Allon Duenhas} rogerioduenhas@utfpr.edu.br Paraná. Curitiba. Paraná. Brasil.

\section{RESUMO}

Este artigo pretende identificar se as cidades inteligentes então contribuindo para o desenvolvimento de cidades sustentáveis. A pesquisa se concretizou numa revisão integrativa de literatura, com base nos termos "cidade inteligente" e "cidade sustentável", realizada nas bases indexadas no Portal de Periódicos da CAPES que atendessem aos critérios: artigos, em português ou inglês, publicados no ano de 2014 a 2018, revisados por pares, que expusessem em sua discussão ponderações sobre cidades inteligentes e cidades sustentáveis. A busca inicial resultou em 80 artigos, que após passar pelos critérios de inclusão e exclusão, resultaram em 07 artigos inclusos à revisão. Os resultados apontam, inicialmente, pela necessidade de amadurecimento dos conceitos de cidade inteligente e cidade sustentável. Há indícios de carência de indicadores para uma aferição realista do desenvolvimento sustentável em cidades inteligentes. O uso de TIC parece ser o caminho mais indicado no alcance de cidades sustentáveis, porém a efetividade dessas ações ainda é questionável. Para trabalhos futuros sugere-se que aprofundem no estudo de metodologias de avaliação de indicadores de sustentabilidade em cidades inteligentes; que se apresentem propostas de construção ao conceito de "cidades inteligentes sustentáveis"; e que aborde o papel do cidadão no desenvolvimento de "cidades inteligentes sustentáveis".

PALAVRAS-CHAVE: Cidade Inteligente; Cidade Sustentável; Desenvolvimento. 


\section{INTRODUÇÃO}

$\mathrm{Na}$ atualidade, as cidades são concentradoras de grande parte da população, da riqueza e das atividades econômicas. Simultaneamente, são os lugares onde mais se encontram problemas sociais e ambientais, com impacto na qualidade de vida de seus usuários.

O modelo de ocupação extensivo do território urbano tem causado problemas de eficiência e sustentabilidade, que retorna como um grande desafio para as políticas de desenvolvimento urbano da atualidade (PORTUGAL, 2015). A compreensão de que providências urgentes devem ser tomadas para reagir às externalidades ambientais negativas - poluição do ar, solo, rios e outros - de maneira séria e concreta resultou na redescoberta da necessidade de práticas mais ecológicas.

Com essa premissa, temos visto nos últimos anos uma proliferação de iniciativas de cidades inteligentes, como meio para estimular as cidades a melhorarem seu desempenho nos três pilares da sustentabilidade: ambiental, econômico e social (MARSAL-LLACUNA, 2016). Muitas das administrações das cidades vêem nos sistemas de Tecnologia da Informação e Comunicação - TIC, aplicações e sistemas de tecnologia urbana inteligentes como potenciais veículos para lidar com seus desafios atuais e futuros de desenvolvimento.

No entanto, a dinâmica das cidades inteligentes é diferenciada das cidades sustentáveis, já que é pelo uso de tecnologias inteligentes que se pauta para alcançar a sustentabilidade. A definição de cidade inteligente é tida como uma visão, manifesto ou promessa com o objetivo de constituir a forma de cidade sustentável e ideal do século XXI (TRINDADE et al., 2017).

Diante dos diversos e imprecisos conceitos, até por ser uma temática relativamente nova, para este artigo se adotou como conceito para cidade inteligente:

Uma cidade inteligente se forma quando investimentos em capital humano e social e tradicional (transporte) e moderna (TIC) infraestruturas tecnologias de comunicação alimentam um crescimento econômico sustentável e qualidade de vida, com uma gestão sábia dos recursos naturais por meio de uma governança participativa (CARAGLIU, DEL BO, NIJKAMP, 2011).

As cidades sustentáveis ficam definidas neste trabalho como:

$$
\begin{aligned}
& \text { “...assentamento humano constituído por uma sociedade com } \\
& \text { consciência de seu papel de agente transformador dos espaços e } \\
& \text { cuja relação não se dá pela razão natureza-objeto e sim por uma } \\
& \text { ação sinérgica entre prudência ecológica, eficiência energética e } \\
& \text { equidade socioespacial” (ROMERO, 2007). }
\end{aligned}
$$

Ambos os conceitos não podem ser considerados como contrastantes; na verdade, eles compartilham muitos pontos em comum. Isso porque a atenção voltada para questões sociais, ambientais e econômicas tem enquadrado o debate sobre sustentabilidade e convergido na definição de cidade inteligente e, obviamente, na noção de cidade sustentável (D'AURIA, TREGUA e VALLEJOMARTOS, 2018).

Segundo Ahvenniemi et al. (2017), "o papel das tecnologias nas cidades inteligentes deve ser permitir o desenvolvimento sustentável das cidades, não na 
nova tecnologia como um fim em si mesmo. Em última análise, uma cidade não é sustentável se não é realmente inteligente ". Em suma, um objetivo geral das cidades inteligentes é melhorar a sustentabilidade com a ajuda de tecnologias.

Ainda que venha sendo objeto crescente de estudo acadêmico nos últimos anos, há poucas evidências empíricas de que, apesar da promessa, as cidades inteligentes contribuem para a agenda de sustentabilidade dessas cidades. A fim de apurar esta questão, de saber se cidade inteligente realmente leva a resultados sustentáveis, este estudo se pauta na questão norteadora: Cidades inteligentes estão contribuindo com o desenvolvimento de cidades sustentáveis?

\section{MÉTODOS}

Este estudo foi elaborado sob método de revisão integrativa, que oportuniza a identificação, análise e síntese de conhecimento, contribuindo com resultados científicos ao tema em questão. Esta metodologia permite uma ampla amostra, com a abrangência de estudos experimentais ou não, combinando dados da literatura teórica e empírica, além de possibilitar um vasto leque de propósitos (SOUZA, SILVA e CARVALHO, 2010).

Para instrumentalizar a revisão integrativa, foram seguidas as seis fases definidos por Souza, Silva e Carvalho (2010): elaboração das perguntas norteadoras; amostragem da literatura; coleta de dados; análise crítica dos estudos incluídos; discussão dos resultados; e apresentação da revisão integrativa.

A busca dos estudos ocorreu no período de setembro a outubro de 2018. Como critérios de inclusão dos estudos foram definidos: artigos, em português e inglês, publicados do ano de 2014 a 2018, periódicos revisados por pares, que apresentassem em sua discussão considerações sobre cidades inteligentes e cidades sustentáveis, indexados na base de dados do Portal de Periódicos da Capes. O recurso utilizado na pesquisa foi a expressão "é (exato)" em "Qualquer" parte do artigo, associado aos descritores específicos. Para critério de exclusão dos artigos foi definido: estudos que não atendessem os critérios de inclusão relacionados.

A realização das buscas se deu com a combinação das palavras-chave: cidad* inteligent*, cidad* sustentáv*, smart cit* e sustainable cit*. Na busca, foram identificados inicialmente 80 artigos para leitura exploratória dos títulos e resumos, os quais foram excluídos os títulos repetidos. Destes, foram selecionados 27 para leitura integral. Após a leitura analítica dos artigos, 07 foram selecionados como objeto de estudo, por apresentarem considerações que respondiam à questão norteadora deste trabalho. O Quadro 1 descreve a etapas do processo. 
Quadro 1 - Etapas do processo de obtenção de referências bibliográficas

\begin{tabular}{|c|c|c|c|c|c|}
\hline Base de dados & $\begin{array}{l}\text { Palavras-chave } \\
\text { cruzadas }\end{array}$ & $\begin{array}{c}\text { № de } \\
\text { referências } \\
\text { obtidas } \\
\end{array}$ & $\begin{array}{l}\text { Resumos } \\
\text { analisados }\end{array}$ & $\begin{array}{l}\text { Referências } \\
\text { selecionadas } \\
\text { para análise } \\
\end{array}$ & $\begin{array}{c}\text { Referências } \\
\text { selecionadas para } \\
\text { revisão }\end{array}$ \\
\hline \multirow{4}{*}{ Capes } & $\begin{array}{l}\text { cidad* } \\
\text { inteligent* e } \\
\text { cidad* } \\
\text { sustentáv* }\end{array}$ & 09 & 04 & 01 & 01 \\
\hline & $\begin{array}{l}\text { smart cit*e } \\
\text { sustainable } \\
\text { cit* }\end{array}$ & 66 & 45 & 26 & 06 \\
\hline & $\begin{array}{l}\text { cidad* } \\
\text { inteligent*e } \\
\text { sustainable } \\
\text { cit* }\end{array}$ & 02 & 01 & 00 & 00 \\
\hline & $\begin{array}{l}\text { cidad* } \\
\text { sustentáv*e } \\
\text { smart cit* }\end{array}$ & 03 & 00 & 00 & 00 \\
\hline Total & & 80 & 50 & 27 & 07 \\
\hline
\end{tabular}

Fonte: Elaborado pelo autor (2018).

\section{RESULTADOS}

Os estudos selecionados foram identificados por periódico, ano de publicação, e título, conforme exposto no Quadro 2. Posteriormente foram extraídos os conceitos abordados em cada artigo, e aplicáveis à questão norteadora.

Quadro 2 - Etapas distribuição das referências incluídas na revisão integrativa

\begin{tabular}{|c|c|c|l|}
\hline $\mathbf{N}^{\mathbf{0}}$ & Periódico & Ano e País & \multicolumn{1}{c|}{ Título } \\
\hline 1 & Cities & 2016 & $\begin{array}{l}\text { What are the differences between sustainable and } \\
\text { smart cities? }\end{array}$ \\
\hline 2 & Urban Island Studies & 2016 & $\begin{array}{l}\text { Island Smart Eco-Cities: Innovation, Secessionary } \\
\text { Enclaves, and the Selling of Sustainability }\end{array}$ \\
\hline
\end{tabular}




\begin{tabular}{|c|c|c|l|}
\hline 3 & $\begin{array}{c}\text { International Journal } \\
\text { of Innovation }\end{array}$ & 2017 & $\begin{array}{l}\text { Smart sustainable cities: bibliometric study and } \\
\text { patent information }\end{array}$ \\
\hline 4 & $\begin{array}{c}\text { Field Actions } \\
\text { Science Reports } \\
\text { The journal of field } \\
\text { actions }\end{array}$ & 2017 & $\begin{array}{l}\text { IBM - Building sustainable cities through } \\
\text { partnerships and integrated approaches }\end{array}$ \\
\hline 5 & $\begin{array}{c}\text { Journal of Cleaner } \\
\text { Production }\end{array}$ & 2018 & $\begin{array}{l}\text { Do Brazilian cities want to become smart or } \\
\text { sustainable? }\end{array}$ \\
\hline 7 & $\begin{array}{c}\text { Land Use Policy } \\
\text { Forecasting \& Social } \\
\text { Change }\end{array}$ & 2018 & $\begin{array}{l}\text { Does smart city policy lead to sustainability of } \\
\text { cities? }\end{array}$ \\
\hline & 2018 & $\begin{array}{l}\text { Smart and sustainable? Five tensions in the visions } \\
\text { and practices of the smart-sustainable city in } \\
\text { Europe and North America }\end{array}$ \\
\hline
\end{tabular}

Fonte: Elaborado pelo autor (2018).

\section{DISCUSSÃO}

Da análise do conteúdo dos artigos selecionados, a partir da questão norteadora desta pesquisa, emergiram as contribuições apresentadas que passam a ser expostas, considerando o trabalho publicado:

No artigo "What are the differences between sustainable and smart cities?" Ahvenniemi et al. (2016) considera uma alteração na avaliação da sustentabilidade para os objetivos da cidade inteligente no século 21. Foram analisados 958 indicadores, divididos em três categorias e 12 setores, em 16 conjuntos de estruturas (oito cidades inteligentes e oito quadros de avaliação da sustentabilidade urbana). Os resultados indicam que as cidades inteligentes apresentam foco mais acentuado em tecnologias modernas e "inteligentes" em comparação com estruturas de sustentabilidade urbana. Também há uma indicação de carência em indicadores ambientais para cidades inteligentes, em detrimento de aspectos sociais e econômicos. O hiato atual entre a cidade inteligente e as estruturas de cidades sustentáveis inspira pela necessidade de melhor desenvolver as cidades inteligentes para também se tornem sustentáveis, a ponto dos autores recomendarem a adoção do termo "cidades inteligentes sustentáveis" em vez de cidades inteligentes. Por fim, recomendam que a avaliação do desempenho de cidades inteligentes não deve se pautar apenas indicadores de resultados que medem a eficiência da implantação de soluções 
inteligentes, mas também indicadores de impacto que medem a contribuição para as metas finais, como sustentabilidade ambiental, econômica ou social.

No artigo "Island Smart Eco-Cities: Innovation, Secessionary Enclaves, and the Selling of Sustainability" Grydehøj e Kelman (2016) abordam cidades inteligentes, cidades ecológicas e cidades sustentáveis ("eco-cidades inteligentes") construídas em pequenas ilhas ou delimitadas por espaço urbano circundante. Para os autores, essas características de espacialidade específicas apresentam benefícios para a venda de cidades ecológicas inteligentes como modelos de inovação sustentável: facilidade de criar valor, facilidade de medir a sustentabilidade e facilidade de comunicar o sucesso. Porém, no entendimento dos autores, esses são aspectos ilusórios, que contribuem para o surgimento de uma sustentabilidade meramente econômica. Essa prática de monetarização do meio ambiente através de serviços ecossistêmicos, incentivando projetos e arquitetura "verdes" em grande parte simbólicos, tira a atenção de práticas insustentáveis em outros lugares e exacerba a desigualdade social, contribuindo para um mundo menos sustentável. Há um privilégio aos interesses corporativos em detrimento dos interesses públicos a paritr do argumento de desenvolvimento sustentável. No entanto, esses benefícios são ilusórios quando se trata de progressos em direção à sustentabilidade. Ainda que sejam bons para proteger a inovação, não significa que todos os usos inovadores sejam igualmente bons para o mundo, já que muitas vezes negligencia muitos tópicos sociais, como espaços de gênero, guetização, acessibilidade para pessoas com deficiências cognitivas e intelectuais, e talvez mais notavelmente os desafios sociológicos de viver em espaços pequenos e densos. Por fim, é necessário reconhecer aquela alta densidade urbana (que geralmente não é um atributo de eco-cidades inteligentes ilhadas, mas que é um atributo de muitas ilhas urbanas) propicia a maior sustentabilidade global, já que os custos e os valores dos serviços ambientais decorrentes dessa densidade são amplamente externalizados. Esforços para reduzir o consumo de recursos dentro das cidades existentes e densificar áreas urbanas de forma sustentável podem não ser tão visíveis ou comercializáveis quanto uma icônica eco-cidade de alto nível, mas seria uma grande tragédia se o potencial de sustentabilidade genuína de desenvolvimento fosse perdido. Por fim, ainda que os exemplos e análises do trabalho demonstrem que as eco-cidades inteligentes não estão totalmente desprovidas de valor inovador ambiental, tecnológico e sociológico, seu verdadeiro valor é às vezes pautado apenas como um diferencial competitivo para alicerçado em demanda consumistas.

No artigo Cidades inteligentes e sustentáveis: estudo bibliométrico e de informações patentárias, Kobayashi et al. (2017) indicam a existência de lacuna teórica na conceituação e caracterização de Cidades Inteligentes e Cidades Sustentáveis. O estudo aponta o quão jovem são as termologias, e que a definição imprecisa e em mutação é uma dificuldade na temática. Observa-se que, a partir da comparação dos conceitos de Cidade Inteligente e Cidade Sustentável, uma cidade pode ser inteligente sem ser sustentável, ou pode ter desenvolvimento sustentável sem uso de Tecnologias de Informação e Comunicação (TIC). Em contraponto, há a apresentação de um conceito para Cidades Inteligentes e Sustentáveis (CIS), como territórios que utilizam Tecnologias de Informação e Comunicação e práticas de desenvolvimento urbano sustentável como meio para buscar a melhoria da qualidade de vida dos cidadãos. Os autores ressaltam que com o uso das TIC e inovações, a gestão da mobilidade urbana sustentável pode ser uma das soluções para se evitar a entropia das cidades, tendo um 
planejamento urbano com ênfase no transporte público ativo e uso de veículos com energia limpa.

No artigo "IBM - Building sustainable cities through partnerships and integrated approaches" Philippe Sajhau (2017) propõe redefinir a cidade inteligente, de modo a melhor compreender a crescente urbanização e a necessidade de adotar um desenvolvimento sustentável. Exemplos como Songdo, na Coréia do Sul: criado do zero em 2003, era para ser um dos projetos de cidade inteligente mais completos do mundo, mas está lutando para atrair moradores. Em contrapartida, áreas já altamente urbanizadas podem acomodar iniciativas de cidades inteligentes. Em cidades européias as dificuldades certamente são maiores - cidades antigas -, mas isso força por soluções mais imaginativas e inovadoras. Para isso, a tecnologia deve atuar como meio e não o fim em si. Vantagens de curto prazo como crescimento econômico regional, economia para a comunidade e benefícios tangíveis aos moradores da cidades - qualidade de transporte, consumo de energia, qualidade de vida, etc. -, além do objetivo do desenvolvimento mais sustentável, devem encorajar autoridades locais a investir nessa área. No contexto, o autor defende o papel da IBM no apoio de projetos de cidades inteligentes, para fornecer conhecimentos em coleta de dados, análise e inteligência. Além das ferramentas tecnológicas e do acesso a uma grande quantidade de informações, a cidade inteligente está inscrita em um projeto inclusivo, focado no desenvolvimento, o que proporciona um papel de destaque aos moradores.

O artigo "Do Brazilian cities want to become smart or sustainable?" de Machado Junior et al. (2018) buscou analisar se os indicadores publicados pelas cidades brasileiras estão alinhados à abordagem de uma cidade inteligente ou sustentável. $O$ estudo indicou que grandes cidades apresentam desempenho superior em relação às médias cidades, e estas superior às pequenas cidades, ainda que em análise individualizada dos indicadores sociais, as pequenas cidades apresentem um conjunto de melhor desempenho. Foi identificado que muitos municípios possuem indicadores ambientais em sua esfera de gestão, em maior ou menor quantidade, e apresentam atenção com a definição de um equilíbrio sustentável entre condições socioeconômicas e ambientais. Porém, o maior destaque para indicadores socioeconômicos - sociais e de tecnologias de informação e comunicação - demonstra foco de atenção das cidades brasileiras no conceito de cidade inteligente, ou seja, o conceito de cidade inteligente pode ser aproximado aos conceitos de cidade sustentável. Os resultados ainda indicaram a carência de indicadores e falta de conceitos que explorem as questões ambientais, o que impede sistematizar uma compreensão da cidade sustentável para os municípios brasileiros.

O estudo "Does smart city policy lead to sustainability of cities?" de Yigitcanlar e Kamruzzaman (2018) avaliam práticas de cidades inteligentes do Reino Unido para investigar suas contribuições para alcançar resultados urbanos sustentáveis. Foram investigadas as mudancas no nível de emissões de dióxido de carbono em 15 cidades de diferentes níveis de "inteligência" no período de 2005 a 2013. Os resultados sugerem que, no contexto das cidades investigadas no Reino Unido, não há fortes evidências sobre: a) uma correlação positiva entre adoção de tecnologia e resultados sustentáveis, e; b) o impacto da "inteligência" da cidade na mudança de emissões de $\mathrm{CO} 2$ ao longo do tempo. Isso indica que, ao menos durante o período do estudo, as práticas de cidades inteligentes no Reino Unido não corresponderam a uma contribuição considerável para a agenda da 
sustentabilidade enfatizando a necessidade de desenvolver agendas de cidades inteligentes que forneçam resultados sustentáveis.

No artigo "Smart and sustainable? Five tensions in the visions and practices of the smart-sustainable city in Europe and North America" Martin, Evans e Karvonen (2018) apresentam uma análise da sustentabilidade inteligente, considerando as críticas estabelecidas da política e das visões da cidade inteligente, juntamente com evidências relativas às experiências reais de iniciativas de cidades inteligentes. Por meio de uma revisão sistemática de literatura de 32 estudos empíricos de cidades pertencentes à Europa e América do Norte, os autores identificaram cinco tensões-chave entre as visões de cidades inteligentes e os objetivos do desenvolvimento urbano sustentável. Os resultados da análise indicaram que, na prática, as iniciativas de cidades inteligentes corroboram com consumismo e com formas insustentáveis de crescimento econômico, negligenciando a equidade social e a sustentabilidade ambiental, deixando de lado as ideias de crescimento verde. Os autores concluem que é o potencial para capacitar e incluir cidadãos representa a chave para desbloquear formas de desenvolvimento urbano inteligente e sustentável que enfatizam a proteção ambiental e a eqüidade social, em vez de meramente reforçar formas neoliberais de desenvolvimento urbano.

O exame dos artigos permitiu a extração alguns pontos ponderantes, que merecem destaque:

a. Indicação de que, para os autores Ahvenniemi et al. (2016), se faz necessária a adoção de conceito de "cidades inteligentes sustentáveis", como um misto de cidades que realmente buscam atingir os dois conceitos síncronamente;

b. Limitação de indicadores ambientais capazes de aferir esse aspecto com precisão em cidades inteligentes. Esse entrave foi frequentemente citado, apontando pela dificuldade na mensuração da sustentabilidade atingida por meio do uso de TIC;

c. Cidades inteligentes tem apresentado foco mais acentuado em tecnologias modernas e "inteligentes" em comparação com estruturas de sustentabilidade urbana, como aponta Ahvenniemi et al. (2016);

d. Indícios de que, em alguns cenários, sustentabilidade ambiental tem sido usada meramente como diferencial comercial, no intuito de atingir êxito econômico. Essas iniciativas de cidades inteligentes corroboram com consumismo e com formas insustentáveis de crescimento econômico;

e. Cidades podem ser inteligentes sem serem sustentáveis, ou ainda podem ter desenvolvimento sustentável sem uso de Tecnologias de Informação e Comunicação (TIC);

f. A chave para atingir o desenvolvimento urbano e sustentável são os cidadãos. A inclusão destes é ponto fundamental;

g. O uso de TIC aparenta ser o caminho que tem sido adotado na busca de se alcançar cidades sustentáveis. Em termos gerais, as cidades inteligentes tem se mostrado o caminho que mais está sendo seguido para se atingir a sustentabilidade ambiental. No entanto, ao que parece, não tem representado uma contribuição consideravel para a agenda da sustentabilidade urbana. 


\section{CONSIDERAÇÕES FINAIS}

Este estudo foi elaborado no esforço de compreender se as cidades inteligentes estão contribuindo para o desenvolvimento de cidades sustentáveis. As cidades inteligentes, pautadas pelo uso de Tecnologia de Informação e Comunicação, são o meio predominante na busca da sustentabilidade urbana. No entanto, as tecnologias "inteligentes" devem ser vistas como um meio, e não o fim em si. A função das tecnologias nas cidades inteligentes deve possibilitar o desenvolvimento sustentável das cidades. Ainda assim, deve se considerar que uma cidade que não é sustentável não é realmente "inteligente".

Os termos atualmente utilizados em maior frequencia "cidade inteligente" e "cidade sustentável" também são usados com ênfase mercadológico, para diferencial competitivo de novas áreas urbanas ou áreas urbanas já existentes. No entanto, apresenta falta de uma abordagem integrada que cubra as preocupações de sustentabilidade.

Outro aspecto relevante deparado pelo autores é quanto aos indicadores de sustentabilidade ambiental, que tem se apresentado como um severo limitante nos quadros dos artigos analisados. As cidades inteligentes não apresentam meios eficazes de mensuração de atendimento aos objetivos sustentáveis. A avaliação da sustentabilidade deve fazer parte do desenvolvimento da cidade inteligente e, assim, integrar a sustentabilidade e estruturas de cidades inteligentes. Portanto, o uso de um termo mais preciso "Cidades inteligentes sustentáveis" (em vez de "cidades inteligentes" ou "cidades sustentáveis") parece ser razoável para que não se negligencie a sustentabilidade em cidades inteligentes.

Diante das considerações, entendemos como relevantes que para estudos futuros se avaliem com maior profundidade metodologias de avaliação de indicadores de sustentabilidade em cidades inteligentes; se apresente uma conceituação, tão precisa quanto possível, para "cidades inteligentes sustentáveis"; o papel do cidadão no desenvolvimento de "cidades inteligentes sustentáveis". 


\title{
Smart cities and sustainable cities: convergence of actions or mere advertising?
}

\begin{abstract}
This article aims to identify whether smart cities then contribute to the development of sustainable cities. The research was carried out in an integrative literature review, based on the terms "smart city" and "sustainable city", carried out on the bases indexed in the CAPES Portal of Journals that met the criteria: articles, in Portuguese or English, published in the year of 2014 to 2018, peer-reviewed, that exposed in their discussion considerations about smart cities and sustainable cities. The initial search resulted in 80 articles, which after going through the inclusion and exclusion criteria, resulted in 07 articles included in the review. The results point, initially, to the need to mature the concepts of smart city and sustainable city. There are indications of a lack of indicators for a realistic assessment of sustainable development in smart cities. The use of ICT seems to be the most indicated way to reach sustainable cities, however the effectiveness of these actions is still questionable. For future work, it is suggested that they deepen the study of methodologies for evaluating sustainability indicators in smart cities; that construction proposals be submitted to the concept of "sustainable smart cities"; and that addresses the role of the citizen in the development of "sustainable smart cities".
\end{abstract}

KEY WORDS: Smart City; Sustainable city; Development. 


\section{REFERÊNCIAS}

AHVENNIEMI, H., HUOVILA, A., PINTO-SEPPÄ, I., e AIRAKSINEN, M. What are the differences between sustainable and smart cities? Cities The International Journal Of Urban POlicy na Planning , 60, 234-245, 2017.

CARAGLIU, A.; DEL BO, C.; NIJKAMP, P. Smart Cities in Europe. Journal of Urban Technology, 18(2), 65-82, 2011.

D'AURIA, Anna; TREGUA, Marco; VALLEJO-MARTOS, Manuel Carlos. Modern Conceptions of Cities as Smart and Sustainable and Their Commonalities. Sustainability, 2018.

GRYDEH $\varnothing J, A ;$ KELMAN, I. Island smart eco-cities: Innovation, secessionary enclaves, and the selling of sustainability. Urban Island Studies, 2, 1-24, 2016.

KOBAYASHI, Andrea Regina Kaneko et al. Smart sustainable cities: bibliometric study and patent information. International Journal of Innovation (IJI), [S.I.], v. 5, n. 1, p. 77-96, feb. 2017.

MACHADO JUNIOR C; NASSIF MANTOVANI RIBEIRO DM; DA SILVA PEREIRA R; BAZANNI R. Do Brazilian cities want to become smart or sustainable? Journal of Cleaner Production, 2018.

MARSAL-LLACUNA, Maria-Lluïsa. City Indicators on Social Sustainability as Standardization Technologies for Smarter (Citizen-Centered) Governance of Cities. Social Indicators Research: An International and Interdisciplinary Journal for Quality-of-Life Measurement, Springer, vol. 128(3), pages 1193-1216, 2016.

MARTIN, Cris J.; EVANS, James; KARVONEN, Andrew. Smart and sustainable? Five tensions in the visions and practices of the smart-sustainable city in Europe and North America. Technological Forecasting and Social Change, Volume 133, 269278, 2018.

PORTUGAL. MINISTÉRIO DO AMBIENTE, ORDENAMENTO DO TERRITÓRIO E ENERGIA. Cidades Sustentáveis 2020. 2015.

ROMERO, Marta A. B.. Frentes do Urbano para a Construção de Indicadores de Sustentabilidade Intra Urbana. Paranoá: cadernos de arquitetura e urbanismo da FAU-UnB, Ano 6, n. 4 (novembro/2007), 2007. 
SAJHAU, Philippe. IBM - Building sustainable cities through partnerships and integrated approaches. Field Actions Science Reports The journal of field actions, Special Issue 16, 2017.

SOUZA, Marcela Tavares; SILVA, Michelly Dias; CARVALHO, Rachel. Revisão integrativa: o que é e como fazer. Einstein, v. 8, n. 1, p. 102-106, 2010.

TRINDADE, E.P.; HINNING, M.P.F.; DA COSTA, E.M. et al. Sustainable development of smart cities: a systematic review of the literature. Journal of Open Innovation: Technology, Market, and Complexity, 2017.

YIGITCANLAR, T.; KAMRUZZAMAN, M. Does smart city policy lead to sustainability of cities? Land Use Policy, 73(1), 49-58, 2018.

Recebido: 12 fev. 2020

Aprovado: 08 abr. 2020

DOI: $10.3895 /$ rbpd.v9n2.10234

Como citar: JUNIOR, M. P.; DUENHAS, R. A. Cidades inteligentes e cidades sustentáveis: convergência de ações ou mera publicidade?. R. bras. Planej. Desenv. Curitiba, v. 9, n. 2, p. 317-328, mai./ago. 2020.

Disponível em: <https://periodicos.utfpr.edu.br/rbpd>. Acesso em: XXX.

Correspondência:

Milton Proença Junior

Av. Sete de Setembro, 3165 - Rebouças - Curitiba, PR

Direito autoral: Este artigo está licenciado sob os termos da Licença CreativeCommons-Atribuição 4.0 Internacional.

\section{(c) (1)}

